\title{
Neutrophil extracellular traps in bronchial aspirates: a quantitative analysis
}

\author{
Shigeto Hamaguchi ${ }^{1,6}$, Tomoya Hirose ${ }^{2,6}$, Naoya Matsumoto², Yukihiro Akeda ${ }^{3}$, \\ Taro Irisawa², Masafumi Seki', Hideo Hosotsubo², Kouji Yamamoto ${ }^{4}$, \\ Osamu Tasaki ${ }^{5}$, Kazunori Oishi ${ }^{3}$, Takeshi Shimazu $^{2}$ and Kazunori Tomono ${ }^{1}$ \\ Affiliations: 'Division of Infection Control and Prevention, Osaka University Graduate School of Medicine, \\ Osaka, ${ }^{2}$ Dept of Traumatology and Acute Critical Medicine, Osaka University Graduate School of Medicine, \\ Osaka, ${ }^{3}$ International Research Center for Infectious Diseases, Research Institute for Microbial Diseases, \\ Osaka University, Osaka, ${ }^{4}$ Dept of Medical Innovation, Osaka University Hospital, Osaka, ${ }^{5}$ Dept of Emergency \\ Medicine, and Unit of Clinical Medicine, Nagasaki University Graduate School of Biomedical Sciences, \\ Nagasaki, Japan. ${ }^{6}$ Both authors contributed equally.
}

Correspondence: K. Tomono, Division of Infection Control and Prevention, Osaka University Graduate School of Medicine, 2-15 Yamadaoka, Suita City, Osaka 565-0871, Japan. E-mail: tomonolahp-infect.med.osaka-u.ac.jp

ABSTRACT Neutrophil extracellular traps (NETs) are structures composed of DNA and granular proteins, which rapidly trap and kill pathogens. The formation of NETs has been detected during infection in animal experiments, but their role in humans is unclear. The purposes of this study were to quantitatively evaluate the production of NETs during acute respiratory infection and to study the relationship between the NET length and various inflammatory mediators.

We examined bronchial aspirates collected from nine intubated patients in an intensive care unit. Samples were collected at the onset of acute respiratory infection (day 0 ) and on days $1,3-5$, and 6-8. The NET length was visualised by immunohistochemistry and quantified using computer tracing software.

The NET length was measured and compared at each time point. The length differed significantly between time points $(\mathrm{p}<0.001)$. NETs were significantly longer on day 1 than on day $0(\mathrm{p}<0.001)$. Neutrophils released NETs abundantly in response to respiratory infection and regression analysis showed that NET length correlated with six clinical parameters (white blood cells, platelets, lactate, CXC ligand-2, interleukin-8, and procalcitonin) as the explanatory variables.

NETs in bronchial aspirates may reflect disease progression of respiratory infections. Quantification of NETs in bronchial aspirates may provide a new indicator of inflammation.

@ERSpublications

NET length increases with respiratory inflammation which correlates with progression of infections http://ow.ly/sPbEX

This article has supplementary material available from www.erj.ersjournals.com

Received: Aug 112013 | Accepted after revision: Dec 292013 | First published online: March 62014

Support statement: This work was supported by a Grant-in-Aid for scientific research from the Ministry of Education, Culture, Sports, Science, and Technology, Japan (No. 21390163 and No. 25293366) and ZENKYOREN (National Mutual Insurance Federation of Agricultural Cooperatives).

Conflict of interest: None declared.

Copyright @ERS 2014 


\section{Introduction}

A patient admitted to an intensive care unit (ICU) sometimes acquires a critical infectious disease such as ventilator-associated pneumonia or sepsis [1]. To date, the therapeutic strategies for treating respiratory infection focus on early diagnosis [2]. To detect respiratory infection before the revelation of lung infiltration on a chest radiograph, Gram staining is performed at the bedside. In Gram staining of sputum, in addition to bacteria and neutrophils, numerous fibrous structures in the background are frequently observed. These structures are thought to be composed of fibrin. However, we reported that these structures might be neutrophil extracellular traps (NETs) [3].

Neutrophils provide a first line of defence in innate immunity [4]. The mechanisms used by neutrophils to eliminate microbes have historically been known to include phagocytosis, generation of reactive oxygen species (ROS), and degranulation [5]. In 2004, BRINKMANN et al. [6] reported a newly identified neutrophil activity, which they called NETs [6]. NETs are produced by activated neutrophils in response to a variety of pro-inflammatory stimuli including lipopolysaccharide, interleukin (IL)-8, tumour necrosis factor (TNF), and various bacteria and fungi [7-9]. High mobility group box 1 (HMGB-1) was also recently reported to promote the formation of NETs [10]. The main components of NETs are decondensed chromatin, histones, and antimicrobial proteins such as neutrophil elastase, myeloperoxidase, and LL37 [11]. Citrullinated histone $\mathrm{H} 3$ (Cit H3) is also a characteristic feature involved in NET formation in vitro. Citrullination of histone $\mathrm{H} 3$ by peptidylarginine deiminase 4 plays a pivotal role in chromatin decondensation during "NETosis" [7, 12]. Inhibition of peptidylarginine deiminase 4 prevents NET formation [13].

Recently, this novel phenomenon, NETosis, has been researched extensively. However, the significance of NET formation in vivo in clinical conditions is not understood fully. We published a preliminary report showing that bronchial aspirates from patients with acute respiratory infections contain abundant NETs [14].

The purposes of this study were to confirm that the fibrous structures in Gram-stained bronchial aspirates are composed of NETs, to measure changes in the length of NETs with time, to evaluate the citrullination of histone $\mathrm{H} 3$, and to determine if there is a relationship between the NET length and various inflammatory mediators in acute respiratory infection.

\section{Methods}

Sample collection

The outline of this study is described in the online supplementary material.

During the study period, from April 2011 to June 2011, bronchial aspirates samples were collected at the onset of acute respiratory infection (day 0 ), and then on days $1,3-5$, and 6-8. The collection of bronchial aspirates was performed by using an Argyle Suction Catheter with Mucus Trap (Covidien, Mansfield, MA, USA) over the endotracheal tube for deeper-lying sampling. The sample was promptly and gently applied to a glass slide. After drying, one sample immediately underwent Gram staining and the other samples were stored at $-80^{\circ} \mathrm{C}$ until the immunohistochemical analysis could be performed.

Acute respiratory infection was diagnosed based on the new onset of purulent endotracheal secretion and the existence of bacterial phagocytosis revealed on a Gram stain of the bronchial aspirate.

\section{Evaluation of clinical background and severity of illness}

Age, sex, body temperature, acute physiological and chronic health evaluation (APACHE II) score, and sequential organ failure assessment (SOFA) score were recorded at the time of admission. Blood samples were collected at each time point when the bronchial aspirates samples were collected and were analysed for laboratory data. The following parameters were measured: white blood cell (WBC) count, platelet count, the concentration of C-reactive protein (CRP), D-dimer, lactate, procalcitonin (PCT), TNF- $\alpha$, IL-6, IL-8, CXC ligand (CXCL)2, HMGB-1, and E-selectin (refer to online supplementary methods).

\section{Identification of NETs}

To identify NETs, we used immunostaining to visualise the major NET components: DNA, neutrophil elastase, and histone $\mathrm{H} 3$. Cit $\mathrm{H} 3$ was also evaluated as a predictor of NET formation by immunohistochemistry (refer to online supplementary methods). Gram staining was conducted using a standard method.

\section{Quantification of NETs}

NET length was quantified using Neurolucida (MBF Bioscience, Williston, VT, USA), software for neuron mapping. NETs were depicted graphically as white lines (fig. 1b). The neutrophil cell bodies (yellow, fig. 1b) were excluded from the calculation of NET length. NET length was defined as the mean length of 

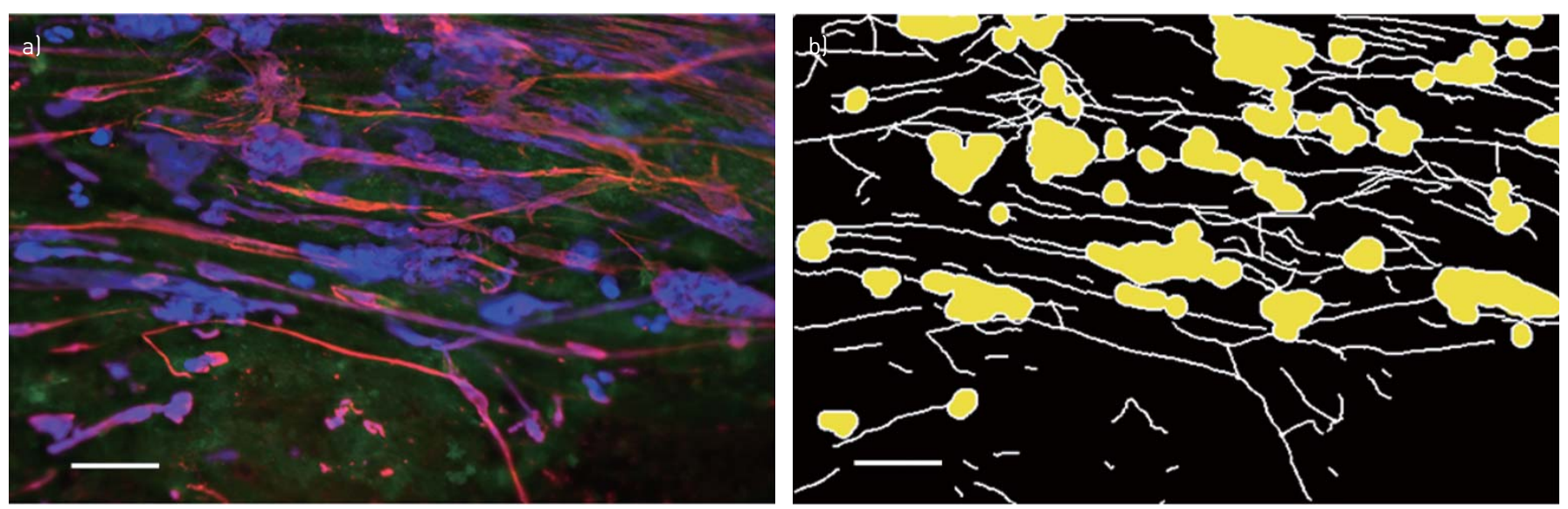

FIGURE 1 Representative adapted images of neutrophil extracellular traps (NETs). a) Analysed using microscopy or b) acquired using Neurolucida (MBF Bioscience, Williston, VT, USA), computer software for tracing neural networks. To calculate the length of NETs, all components merged in a fluorescent picture were traced by the software and each aggregate was excluded from calculation as an actual cell body. Yellow circle: cell body; white line: NETs. Scale bars $=30 \mu \mathrm{m}$.

NETs calculated by dividing the total length of the white line in the graphical depiction by the total number of white lines.

\section{Statistical analysis}

Continuous variables are presented as median and interquartile range (IQR). Fisher's exact test and t-test were used to identify differences between time points in the study group. Single- and multiple-regression analyses were used to identify associations between NET length and biological parameters. All statistical analyses were performed using SAS software (SAS Institute Inc., Cary, NC, USA) for regression analyses and Prism software (version 5.02; GraphPad Software, San Diego, CA, USA).

\section{Results}

\section{Identification of NETs in Gram-stained bronchial aspirates}

To clarify whether the fibrous structures in bronchial aspirates are indeed NETs, we evaluated purulent bronchial aspirates using both Gram stain and immunostain on the same slide. As shown in figure 2, almost all fibres detected in Gram staining also stained simultaneously with 4,6-diamidino-2-phenylindole (DAPI) and neutrophil elastase. This result indicates that most of these fibrous structures observed using Gram stain were NETs.

\section{Characteristics of the study participants}

In this study, we evaluated the production of NETs in bronchial aspirates at each time point based on our protocol. During the study period, 263 patients were admitted to the ICU, and 49 of the 263 patients were intubated. 40 patients were excluded according to the exclusion criteria, details of which are given in the online supplementary material. The final number of patients included in the study was nine.

The characteristics of the patients are shown in table 1 . The study group comprised of six males and three females with a median age of 63.0 years (IQR, 52.0-75.0 years). The major diagnoses for hospitalisation were trauma $(n=2)$, resuscitated cardiopulmonary arrest $(n=3)$, peritonitis $(n=1)$, gas gangrene $(n=1)$, bacterial meningitis $(n=1)$, and pneumonia $(n=1)$. At the time of admission, the median body temperature was $37.6^{\circ} \mathrm{C}$ (IQR $36.8-38.2^{\circ} \mathrm{C}$ ), the median SOFA score was 7.0 (IQR 4.0-10.0), and the median APACHE II score was 21.0 (IQR 21.0-25.0). Seven out of the nine patients survived; two patients died during their ICU stay.

\section{Variation in the appearance of NETs over time}

To detect the NETs in the bronchial aspirates, DNA and histone H3 were visualised simultaneously using immunostaining. NETs were recognised as the merged fibrous structures using these components (fig. 3a).

Previously, we reported that the appearance of NETs in bronchial aspirates increased gradually with the duration and severity of infection and decreased with improvement in the patient's condition [14]. Likewise, the immunohistochemical images of a representative case are shown in figure 3. Only a few NETs were identified in the sample on day 0 , the onset of acute respiratory infection. On day 1 , we observed infiltration of neutrophils and abundant expanse of NETs. After treatment with appropriate antibiotics, the 

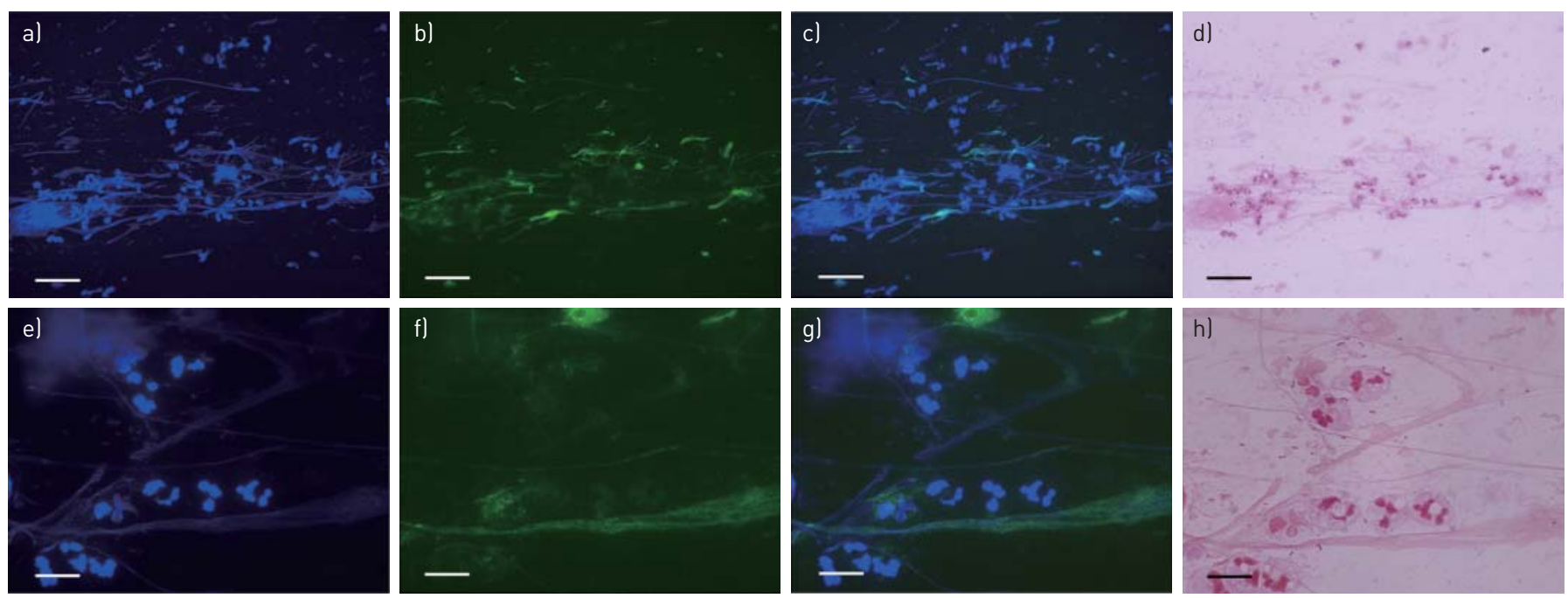

FIGURE 2 Representative images of immunostaining ( $\mathrm{a}-\mathrm{c}$ and $\mathrm{e}-\mathrm{g}$ ) and Gram staining (red, $\mathrm{d}$ and $\mathrm{h}$ ) of bronchial aspirates in the same sample. Neutrophil extracellular traps were detected as co-localised fibre with 4,6-diamidino-2-phenylindole (blue, $a$ and e) and neutrophil elastase (green, $b$ and $\mathrm{f}$ ) by immunohistochemistry; they are identical to the fibrous structures delineated using the Gram stain. Images c) and g) are merged images of a) and b), and e) and f), respectively. a-d) Scale bars $=50 \mu \mathrm{m}$ and $\mathrm{e}-\mathrm{f}$ ) scale bars $=10 \mu \mathrm{m}$.

respiratory infection improved gradually. In association with the recovery, the NET length shortened, and the numbers of NETs decreased gradually by days 5 and 7 .

\section{Citrullination of histone $\mathrm{H} 3$ before NET formation}

We observed Cit $\mathrm{H} 3$ simultaneously to identify the preliminary phase of NET formation. A representative case is shown in figure 3. Cit $\mathrm{H} 3$ was detected in the nucleus of a subset of neutrophils on day 0 even though these neutrophils did not extrude fibrous NETs (white arrowhead in fig. 3). On day 1, the detected area of Cit H3 spread extensively inside the nucleus in some cells, and Cit H3 was detected in parts of the released NETs (white arrow in fig. 3). In general, neutrophils and NETs containing Cit H3 were rarely observed on day 5 or later (fig. 3).

\section{Quantification of NETs by length}

The NET length was measured using the Neurolucida software program to quantify the change in the NET length. The mean NET lengths at each time point (days $0,1,3-5$, and 6-8) were compared. Significant changes were identified during the clinical course by analysis of variance for repeated measures $(\mathrm{p}<0.001$; fig. 4). Tukey's post hoc multiple comparison test showed that the NET length was significantly longer on day 1 than on day $0(\mathrm{p}<0.001)$ and that the NET length was significantly shorter on days $6-8$ than on day 1 $(\mathrm{p}=0.0132)$.

To confirm the identification of the filamentous structure as NETs, we further analysed how the length of this structure changed over time and how it degrades with DNase I in live samples with SYTOX orange (Invitrogen, Carlsbad, CA, USA), a cell membrane impermeant nucleic acid stain, by time-lapse imaging

TABLE 1 Characteristics of study participants

\begin{tabular}{|c|c|c|c|c|c|c|c|}
\hline Patient & Age years & Sex & Condition & Body temperature ${ }^{\circ} \mathrm{C}$ & SOFA score & APACHE II score & Survived \\
\hline 1 & 63 & Male & Trauma & 38.2 & 2 & 6 & Yes \\
\hline 2 & 82 & Male & Cardiac arrest & 37 & 7 & 21 & Yes \\
\hline 3 & 52 & Female & Trauma & 38.9 & 9 & 21 & Yes \\
\hline 4 & 41 & Female & Meningitis & 38.1 & 4 & 6 & Yes \\
\hline 5 & 10 & Male & Cardiac arrest & 36.3 & 11 & 28 & Yes \\
\hline 6 & 78 & Female & Pneumonia & 36.4 & 4 & 22 & Yes \\
\hline 7 & 73 & Male & Gas gangrene & 36.8 & 10 & 25 & Yes \\
\hline 8 & 75 & Male & Peritonitis & 38.3 & 5 & 27 & No \\
\hline 9 & 56 & Male & Cardiac arrest & 37.6 & 10 & 21 & No \\
\hline
\end{tabular}

SOFA: sequential organ failure assessment; APACHE II: acute physiological and chronic health evaluation. 

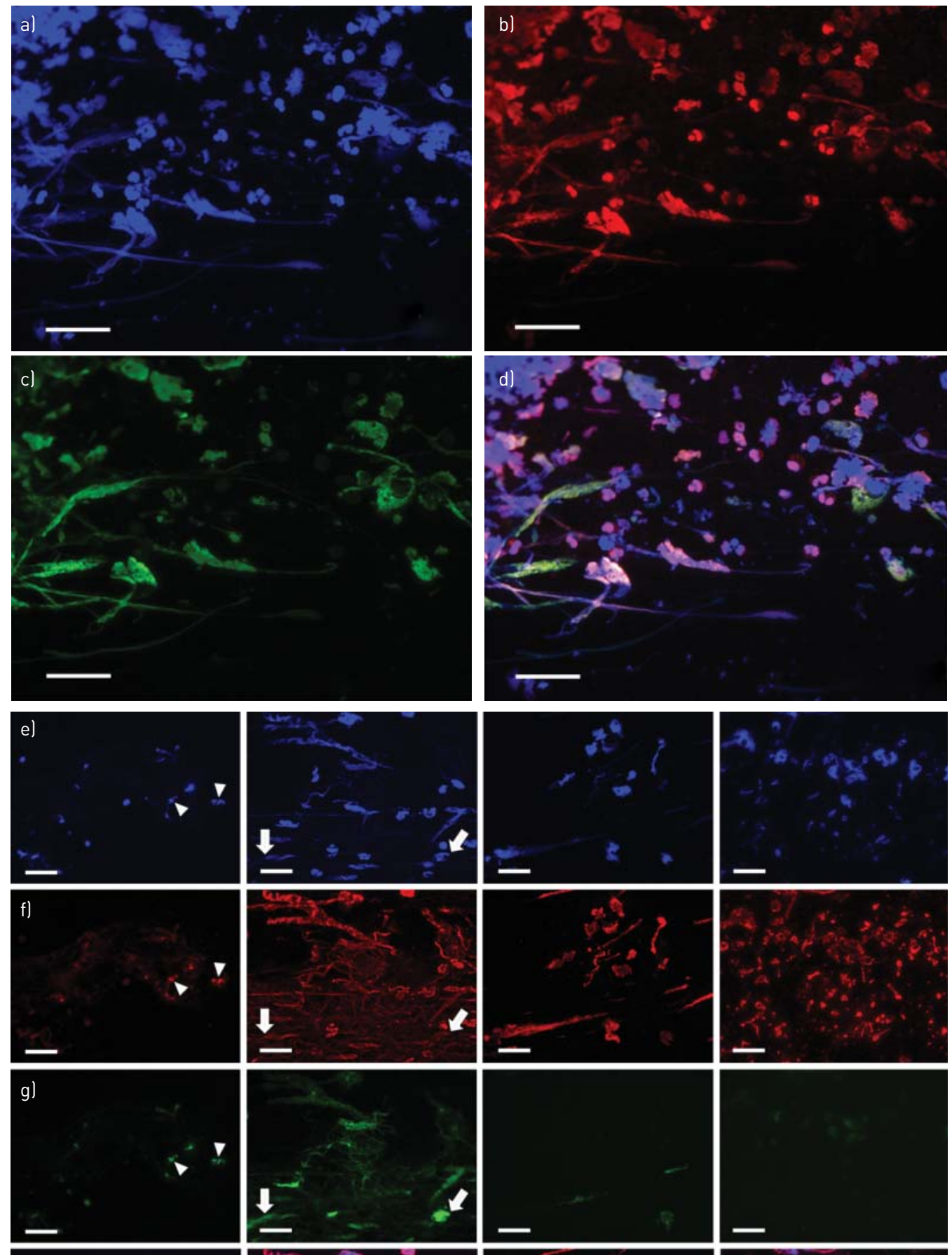

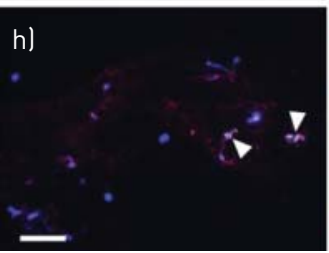

Day 0

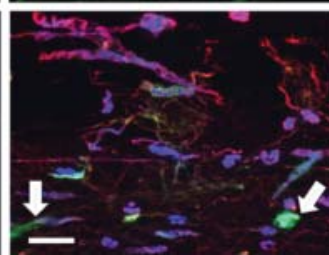

Day 1

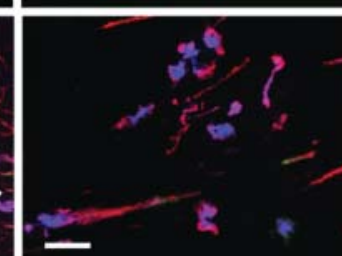

Day 5

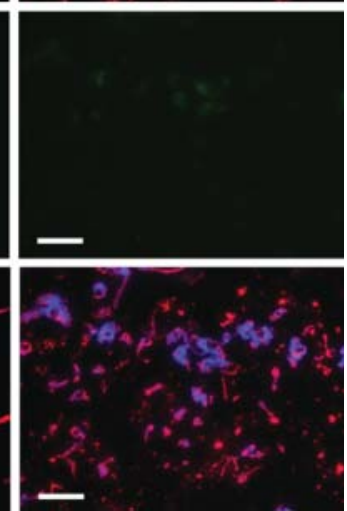

Day 7

FIGURE 3 Representative time course images for the immunohistochemical staining of bronchial aspirates in the same patient. Single stain images for a) 4,6-diamidino-2-phenylindole (DAPI), b) histone H3, c) citrullinated histone H3 (Cit H3), and d) their merged image. Neutrophil extracellular traps (NETs) are recognised as fibrous structures with these components. e- $\mathrm{h}$ ) Representative images of time-dependent changes using e) DAPI f) histone H3, and g) Cit H3 to show appearance of NETs related to disease progression of respiratory infection from day 0 to day 7 . h) The merged image of e-g for each day. White arrowhead: Cit $\mathrm{H} 3$ detected in the nucleus; white arrow: Cit $\mathrm{H} 3$ on NETs. Scale bars $=30 \mu \mathrm{m}$. 


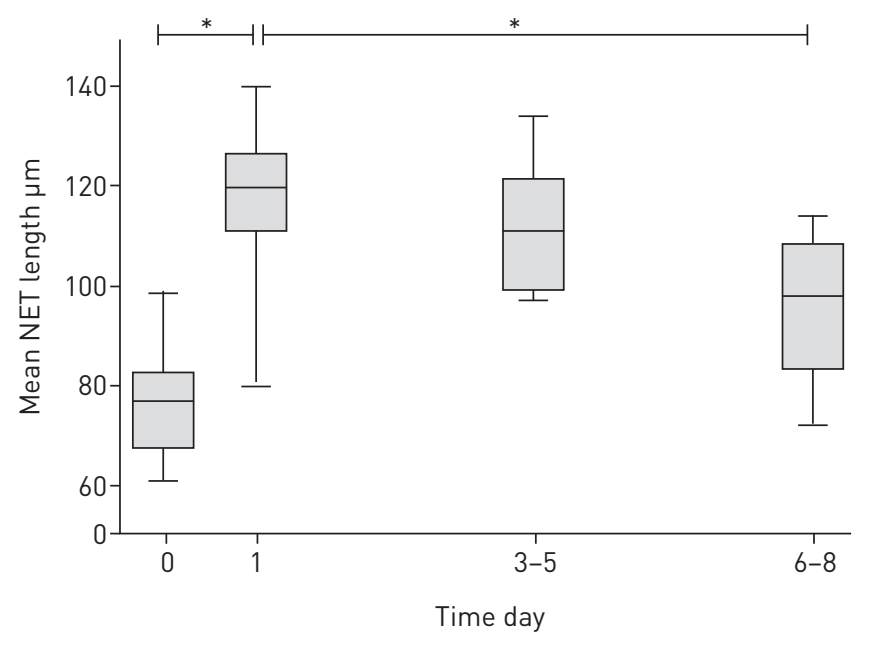

FIGURE 4 Time-dependent changes in mean neutrophil extracellular trap (NET) length in bronchial aspirates evaluated by Neurolucida software (MBF Bioscience, Williston, VT, USA). The changes in mean NET length during the clinical course of infection between day 0 and day 1 , and between day 1 and day $6-8$ were significant. ${ }^{*}: \mathrm{p}<0.05$, ANOVA for repeated measure was $\mathrm{p}<0.001$.

over a 0.5 -h period. As a result, filamentous NETs were gradually stretched along with the stream of bronchial aspirate (online supplementary video 1, arrow head). Furthermore, compared with control (online supplementary video 2), treatment of bronchial aspirates with DNase I results in the gradual shortening and melting of the filamentous NETs (online supplementary video 3 ).

\section{The relationships between NET length and clinical parameters}

To evaluate the associations between NET length and systemic inflammation, we analysed the relationships between the mean NET length and clinical parameters and inflammatory cytokine levels in serum. The data for each time point are shown in table 2. The relationships between NET length and the following parameters were assessed: body temperature, SOFA score, APACHE II score, WBC and platelet count, blood concentration of lactate, and serum concentration of C-reactive protein, D-dimer, PCT, tumour necrosis factor- $\alpha$, IL-6, IL-8, CXCL-2, HMGB-1, and E-selectin.

We investigated these associations by multiple regression analysis to confirm whether NET length could be predicted by present or previous clinical parameters. A multiple regression model was constructed with the NET length as the dependent variable and clinical data as independent variables. The multiple regression analysis demonstrated that the regression model with dependent and independent variables on day 1 was only meaningful because the F-tests for the model rejected the hypothesis of joint nonsignificance of the independent variables. Scatter plots of all clinical parameters and NET length on day 1 are shown (fig. 5). In

TABLE 2 Clinical parameters, serum inflammatory cytokine concentrations, and neutrophil extracellular traps (NETs) length in bronchial aspirates at each time point

Parameter Day

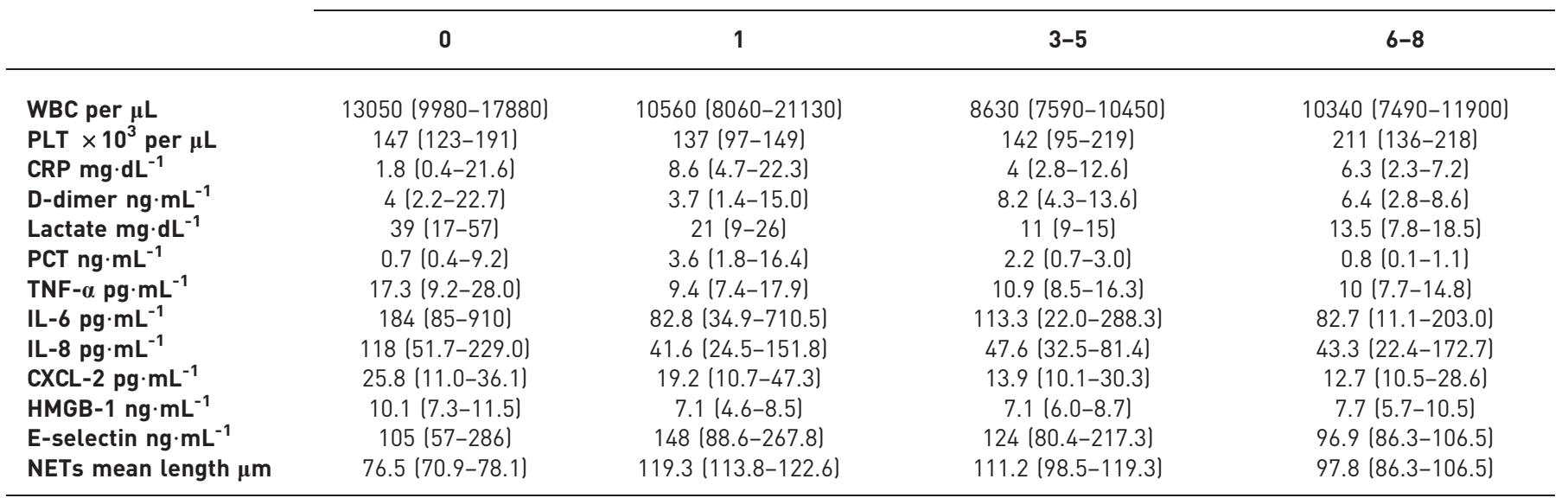

Data are presented as median (interquartile range). WBC: white blood cells; PLT: platelet; CRP: C-reactive protein; PCT: procalcitonin; TNF: tumour necrosis factor; IL; interleukin; CXCL: CXC ligand; HMBG-1: high-mobility group box-1. 


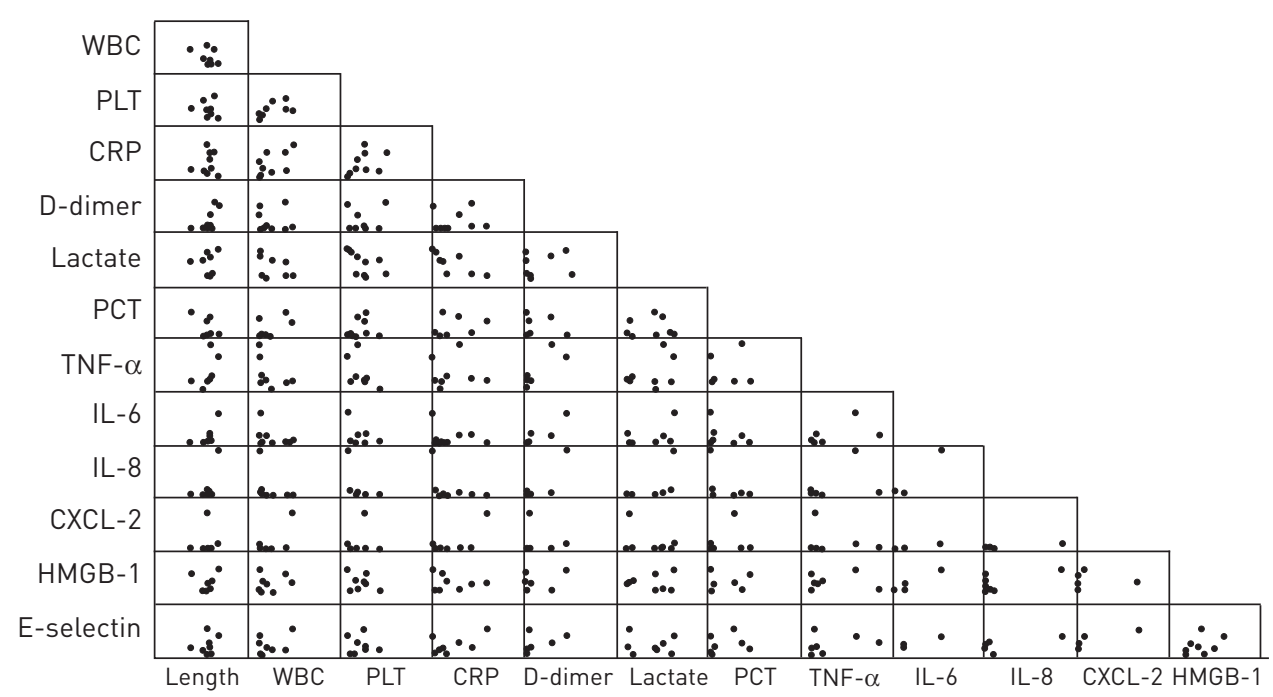

FIGURE 5 Scatter plots of each clinical parameter and mean neutrophil extracellular trap (NET) length as independent variables on day 1 . None of these independent variables showed a significant difference. WBC: white blood cells; PLT: platelet; CRP: C-reactive protein; PCT: procalcitonin; TNF: tumour necrosis factor; IL; interleukin; CXCL: CXC ligand; HMBG-1: high-mobility group box-1.

the regression model, six parameters (WBC and platelet counts, and concentrations of CXCL-2, IL-8, lactate, and PCT) were selected using a stepwise method (table 3). NET length on day 1 correlated with these six inflammatory markers on day 1 . The estimated regression model with these six parameters was as follows: mean NET length $=-1.23 \times$ WBC count $+0.88 \times$ CXCL $-2+0.44 \times$ IL- $8-0.29 \times$ lactate $+0.18 \times$ platelet count $-0.06 \times$ PCT.

\section{Discussion}

The Gram stain is widely used in clinical-infection diagnostics because of its simplicity. In the ICU of our hospital, Gram staining is performed on a routine basis at bedside. Through this experience, we noticed the presence of numerous fibrous structures in the bronchial aspirate samples from patients with acute respiratory infection and that the amount of fibres fluctuated over time. We previously demonstrated for the first time that NETs could be detected immunohistochemically even after Gram staining in the same smear sample [3]. In this study, we used the same method to demonstrate definitively that these fibrous structures seen in Gram staining are composed of NETs (fig. 2). This observation suggests that NETs can be identified using Gram staining alone without the need for immunostaining. This finding will be of great value to the clinician because it means that, along with the identification of type and propagation status of bacteria, evaluation of NET appearance on Gram-stained samples alone can provide information about the biological response to infection without the need for time-consuming procedures.

The quantitative analysis of NET length showed that NET length in bronchial aspirates changed dynamically during the course of respiratory infection. Neutrophils released abundant NETs into bronchial aspirates in response to acute respiratory infections, after which the amount of NETs decreased and the fibres became fragmented as the infection subsided (fig. 3).

Although the mechanism of varying NET length is uncertain, it is known that DNase I exists and has some function in sputum [15]. Our data suggested that exposure to DNase I mainly results in shortening of NETs (online supplementary video 3). It is possible that nascent production of NETs from neutrophils mainly results in extension of existing NETs. A flow of bronchial secretion in the airway might play a part in increasing NET length (online supplementary video 1), as evidenced by some reports showing that DNA was stretched by the traction force $[16,17]$. Thus, it is conceivable that decreasing NET length indicates termination of bronchial infection because of a lack of new production of NETs in bronchi resulting from the resolution of infection.

Researchers have tried to quantify NETs in vivo. However, quantification of NETs in vivo is more difficult than expected because NETs are an extracellular fibrous component, which is difficult to measure precisely by commonly used experimental techniques such as Western blotting, ELISA, PCR, or flow cytometry. Some studies have reported on using the quantity of circulating free DNA to estimate the quantity of NETs in serum $[18,19]$. However, it is uncertain whether circulating free DNA reflects the amount of NETs directly. The quantification method we used here is considered to be reliable because the NETs are 
TABLE 3 Results of the multiple regression analysis of variables related to neutrophil extracellular trap length

\begin{tabular}{lcc} 
& Standardised regression coefficient & p-value \\
\hline Intercept & 0 & 0.004 \\
White blood cells & -1.23 & 0.016 \\
CXC ligand-2 & 0.88 & 0.016 \\
Interleukin-8 & 0.44 & 0.029 \\
Lactate & -0.29 & 0.041 \\
Platelet & 0.18 & 0.075 \\
Procalcitonin & -0.06 & 0.199 \\
\hline
\end{tabular}

The value of adjusted $\mathrm{R} 2=0.999$.

visualised morphologically and the length is measured directly on the slides. A disadvantage is that it takes a relatively long time to obtain the result. Therefore, a simpler method to quantify NET appearance in human samples is needed for more practical clinical application.

In the current study, Cit $\mathrm{H} 3$ was detected inside the nucleus in a subpopulation of neutrophils on day 0 , and many neutrophils expressing Cit H3 appeared at the time of maximum NET production on day 1. Citrullination of histones is thought to be the first step of nuclear decondensation [12]. In this research, polymorphonuclear leukocytes containing $\mathrm{Cit} \mathrm{H} 3$ were predominant in the early infection phase, but were observed rarely in the convalescent period. This observation supports the idea that citrullination of histone $\mathrm{H} 3$ is mandatory for the process of NETosis before nuclear decondensation. A previous report suggested that $\mathrm{Cit} \mathrm{H} 3$ is released into the circulation during the early stage of lipopolysaccharide-induced shock and it is associated with the severity of shock in a mouse model [20]. Our data suggests that the quantity of Cit $\mathrm{H} 3$ has potential as a clinical biomarker of the severity of infection, independent from other well-known inflammatory biomarkers such as C-reactive protein and PCT.

The dynamic change in NET length described above correlated with six inflammatory parameters (table 3): WBC and platelet counts, blood concentration of lactate, and serum concentrations of CXCL-2, IL-8, and PCT. These are well-known markers that reflect the biological response to infection. WBC count correlated negatively and most strongly with NET length. In general, WBC count is elevated in the early stages of inflammation. By contrast, we found that the peak of the NET length was 1 day after the onset of infection. We speculate that this gap and the negative correlation between WBC count and NET length reflect the shift of WBCs from circulation to the respiratory tract.

After WBC count, CXCL-2 levels had the second strongest correlation with NET length. Also called MIP-2 or growth-regulated protein (GRO)-beta, CXCL-2 is secreted by monocytes and macrophages, and promotes chemotaxis of neutrophils [21]. The third most strongly correlated parameter was IL-8 concentration, which also belongs to the CXC chemokine family. IL-8 is a pro-inflammatory cytokine produced by macrophages and other cells, such as epithelial cells, and its level increased during communityacquired pneumonia and ventilator-associated pneumonia [22]. It has been reported that IL-8 can trigger the release of NETs in vitro [6]. Both the accumulation of polymorphonuclear leukocytes and the induction of NETs in the respiratory tract might be induced partly by CXCL-2 or IL- 8 produced by resident macrophages in the lung [23], although confirmation is needed.

An increase in blood lactate level reflects peripheral hypoperfusion and is a predictor of patient mortality under septic conditions in the ICU [23]. In this study, lactate level correlated negatively with NET length. ROS species are an essential substance needed for production of NETs [24]. Volume resuscitation and improved oxygenation by initiation of ventilator management in hypoxic conditions should increase the generation of ROS because of abrupt oxidative stress [25]. One possible explanation of the negative correlation between lactate level and NET production is that the amount of ROS might be insufficient to induce NET production during the phase when lactate level increases. The relationship between lactate level and NETs should be explored to elucidate the biological function of NETs in critical illnesses such as circulatory insufficiency and hypoxia.

Platelet count and PCT level also correlated significantly with NET length in the regression analysis. Platelet-neutrophil interactions play a pivotal role in NETosis [26, 27]. PCT is commonly used as an inflammatory marker and is useful for predicting the prognosis and deciding whether to use antibiotics in pneumonia [28, 29]. 
Nosocomial tracheobronchitis is common in mechanically ventilated ICU patients [30]. A recent paper reported that approximately one third of ventilator-associated tracheobronchitis (VAT) patients later developed ventilator-associated pneumonia (VAP) [31]. Our method could potentially be utilised to predict the progression to VAT, which could result in decreased numbers of VAP patients. Our study also suggests that Gram staining can be used to evaluate NETs as a surrogate for immunohistochemical detection.

Limitations of this study are the small number of patients enrolled in this study and that the patient population was heterogeneous. Simple correlations could not been detected between any single inflammatory parameter and the mean NET length, even though there were significant differences in the NET length. Identification of biological parameters related to NET formation will need larger studies with more participants. Additionally, in this study, we did not investigate the relationship between NET length and bacterial number in bronchial aspirates. Elucidating this relationship may promote further understanding of the biological relevance of NET length. Other candidate molecules involved in NET production or clearance should be examined in the blood as well as bronchial aspirates. Further studies will help clarify the biological significance of NETs in bronchial aspirates in various clinical conditions.

In conclusion, our findings suggest that NET length in bronchial aspirates reflects the immunological and inflammatory states associated with disease progression in respiratory infections. NET length in bronchial aspirates might provide a new inflammatory biomarker that could be applied when making decisions about the initiation and cessation of antibiotics or immunomodulatory therapy.

\section{References}

1 Richards MJ, Edwards JR, Culver DH, et al. Nosocomial infections in medical intensive care units in the United States. Crit Care Med 1999; 27: 887-892.

2 Matsushima A, Tasaki O, Shimizu K, et al. Preemptive antibiotic treatment based on Gram staining reduced the incidence of ARDS in mechanically ventilated patients. J Trauma 2008; 65: 309-315.

3 Hamaguchi S, Seki M, Yamamoto N, et al. Case of invasive nontypable Haemophilus influenzae respiratory tract infection with a large quantity of neutrophil extracellular traps in sputum. J Inflamm Res 2012; 5: 137-140.

4 Lekstrom-Himes JA, Gallin JI. Immunodeficiency diseases caused by defects in phagocytes. N Engl J Med 2000; 343: 1703-1714.

Nathan C. Neutrophils and immunity: challenges and opportunities. Nat Rev Immunol 2006; 6: 173-182.

6 Brinkmann V, Reichard U, Goosmann C, et al. Neutrophil extracellular traps kill bacteria. Science 2004; 303: $1532-1535$.

7 Remijsen Q, Kuijpers TW, Wirawan E, et al. Dying for a cause: NETosis, mechanisms behind an antimicrobial cell death modality. Cell Death Differ 2011; 18: 581-588.

8 Fuchs TA, Abed U, Goosmann C, et al. Novel cell death program leads to neutrophil extracellular traps. J Cell Bio 2007; 176: 231-241.

9 Pilsczek FH, Salina D, Poon KK, et al. A novel mechanism of rapid nuclear neutrophil extracellular trap formation in response to Staphylococcus aureus. J Immunol 2010; 185: 7413-7425.

10 Tadie JM, Bae HB, Jiang S, et al. HMGB1 promotes neutrophil extracellular trap formation through interactions with Toll-like receptor 4. Am J Physiol Lung Cell Mol Physiol 2013; 304: L342-L349.

11 Metzler K, Fuchs T, Nauseef W, et al. Myeloperoxidase is required for neutrophil extracellular trap formation: implications for innate immunity. Blood 2011; 117: 953-959.

12 Neeli I, Khan SN, Radic M. Histone deimination as a response to inflammatory stimuli in neutrophils. J Immunol 2008; 180: 1895-1902.

13 Wang Y, Li M, Stadler S, et al. Histone hypercitrullination mediates chromatin decondensation and neutrophil extracellular trap formation. J Cell Biol 2009; 184: 205-213.

14 Hirose T, Hamaguchi S, Matsumoto N, et al. Dynamic changes in the expression of neutrophil extracellular traps in acute respiratory infections. Am J Respir Crit Care Med 2012; 185: 1130-1131.

15 Cantin AM. DNase I acutely increases cystic fibrosis sputum elastase activity and its potential to induce lung hemorrhage in mice. Am J Respir Crit Care Med 1998; 157: 464-469.

16 Gore J, Bryant Z, Nöllmann M, et al. DNA overwinds when stretched. Nature 2006; 442: 836-839.

17 van Mameren J, Gross P, Farge G, et al. Unraveling the structure of DNA during overstretching by using multicolor, single-molecule fluorescence imaging. Proc Natl Acad Sci USA 2009; 106: 18231-18236.

18 Margraf S, Logters T, Reipen J, et al. Neutrophil-derived circulating free DNA (cf-DNA/NETs): a potential prognostic marker for posttraumatic development of inflammatory second hit and sepsis. Shock 2008; 30: 352-358.

19 Altrichter J, Zedler S, Kraft R, et al. Neutrophil-derived circulating free DNA (cf-DNA/NETs), a potential prognostic marker for mortality in patients with severe burn injury. Eur J Trauma Emerg Surg 2010; 36: 551-557.

20 Li Y, Liu B, Fukudome EY, et al. Identification of citrullinated histone $\mathrm{H} 3$ as a potential serum protein biomarker in a lethal model of lipopolysaccharide-induced shock. Surgery 2011; 150: 442-451.

21 Iida N, Grotendorst GR. Cloning and sequencing of a new gro transcript from activated human monocytes: expression in leukocytes and wound tissue. Mol Cell Biol 1990; 10: 5596-5599.

22 Bonten MJ, Froon AH, Gaillard CA, et al. The systemic inflammatory response in the development of ventilatorassociated pneumonia. Am J Respir Crit Care Med 1997; 156: 1105-1113.

23 Trzeciak S, Dellinger RP, Chansky ME, et al. Serum lactate as a predictor of mortality in patients with infection. Intensive Care Med 2007; 33: 970-977.

24 Keshari RS, Verma A, Barthwal MK, et al. Reactive oxygen species-induced activation of ERK and p38 MAPK mediates PMA- induced NETs release from human neutrophils. J Cell Biochem, 2013: 532-540.

25 Kavazis AN, Talbert EE, Smuder AJ, et al. Mechanical ventilation induces diaphragmatic mitochondrial dysfunction and increased oxidant production. Free Radic Biol Med 2009; 46: 842-850. 
Clark SR, Ma AC, Tavener SA, et al. Platelet TLR4 activates neutrophil extracellular traps to ensnare bacteria in septic blood. Nat Med 2007; 13: 463-469.

27 Fuchs T, Brill A, Durschmied D, et al. Extracellular DNA traps promote thrombosis. Proc Natl Acad Sci USA 2010; 107: 15880-15885.

28 Kruger S, Ewig S, Marre R, et al. Procalcitonin predicts patients at low risk of death from community-acquired pneumonia across all CRB-65 classes. Eur Respir J 2008; 31: 349-355.

29 Christ-Crain M, Stolz D, Bingisser R, et al. Procalcitonin guidance of antibiotic therapy in community-acquired pneumonia a Randomized trial. Am J Respir Crit Care Med 2006; 174: 84-93.

30 Nseir S, Di Pompeo C, Pronnier P, et al. Nosocomial tracheobronchitis in mechanically ventilated patients: incidence, aetiology and outcome. Eur Respir J 2002; 20: 1483-1489.

31 Craven DE, Lei Y, Ruthazer R, et al. Incidence and outcomes of ventilator-associated tracheobronchitis and pneumonia. Am J Med 2013; 126: 542-549. 